\title{
Layout preferences concerning matching in a fully differential $\Delta \Sigma$ modulator design
}

\author{
Hakan Binici · Juha Kostamovaara
}

Published online: 26 May 2007

(C) Springer Science+Business Media, LLC 2007

\section{Erratum to: Analog Integr Circ Sig Process DOI 10.1007/s10470-006-9004-x}

The publisher regrets that incorrect data was printed from Table 3 in "Layout Preferences Concerning Matching in a Fully Differential $\Delta \Sigma$ Modulator Design'” by Hakan Binici and Juha Kostamovaara, which published in Analog Integrated Circuits and Signal Processing 50:2, pp. 95-103. The corrected Table 3 is shown below.

The online version of the original article can be found under doi:10.1007/s10470-006-9004-x.

H. Binici $(\square) \cdot J$. Kostamovaara Department of Electrical and Information Engineering and Infotech Oulu, University of Oulu, P.O. Box 4500, 90014 Oulu, Finland

e-mail: hakan.binici@ee.oulu.fi 
Table 3 Measured modulator performance for design B

\begin{tabular}{|c|c|c|c|c|}
\hline & $\begin{array}{l}\text { Normal operation, normal } \\
\text { current }\end{array}$ & $\begin{array}{l}\text { Normal operation, reduced } \\
\text { current }\end{array}$ & $\begin{array}{l}\text { Increased sampling } \\
\text { frequency }\end{array}$ & $\begin{array}{l}\text { Increased oversampling } \\
\text { ratio }\end{array}$ \\
\hline Sampling frequency & $32 \mathrm{kHz}$ & $32 \mathrm{kHz}$ & $250 \mathrm{kHz}$ & $192 \mathrm{kHz}$ \\
\hline Oversampling ratio & 48 & 48 & 48 & 288 \\
\hline Signal bandwidth & $330 \mathrm{~Hz}$ & $330 \mathrm{~Hz}$ & $2.5 \mathrm{kHz}$ & $330 \mathrm{~Hz}$ \\
\hline Supply voltage & $2.8 \mathrm{~V}( \pm 1.4 \mathrm{~V})$ & $2.8 \mathrm{~V}( \pm 1.4 \mathrm{~V})$ & $2.8 \mathrm{~V}( \pm 1.4 \mathrm{~V})$ & $2.8 \mathrm{~V}( \pm 1.4 \mathrm{~V})$ \\
\hline $\begin{array}{l}\text { Minimum supply } \\
\text { voltage }\end{array}$ & $2.2 \mathrm{~V}( \pm 1.1 \mathrm{~V})$ & $2.2 \mathrm{~V}( \pm 1.1 \mathrm{~V})$ & $2.6 \mathrm{~V}( \pm 1.3 \mathrm{~V})$ & $2.6 \mathrm{~V}( \pm 1.3 \mathrm{~V})$ \\
\hline Reference voltage & $1.0 \mathrm{~V}$ & $1.0 \mathrm{~V}$ & $1.0 \mathrm{~V}$ & $1.0 \mathrm{~V}$ \\
\hline Input $\mathrm{CM}$ voltage & $-0.5 \mathrm{~V}$ & $-0.5 \mathrm{~V}$ & $-0.5 \mathrm{~V}$ & $-0.5 \mathrm{~V}$ \\
\hline Peak SNR/SNDR & $74 / 71 \mathrm{~dB}$ & $70 / 68 \mathrm{~dB}$ & $65 / 62 \mathrm{~dB}$ & $78 / 76.5 \mathrm{~dB}$ \\
\hline Max./Min. input levels & $-2 /-73 \mathrm{~dB}$ & $-2 /-73 \mathrm{~dB}$ & $-8 /-69 \mathrm{~dB}$ & $-10 /-88 \mathrm{~dB}$ \\
\hline Dynamic range & $71 \mathrm{~dB}$ & $71 \mathrm{~dB}$ & $61 \mathrm{~dB}$ & $78 \mathrm{~dB}$ \\
\hline Resolution & 12 bits & 11.5 bits & 10 bits & 13 bits \\
\hline PSRR & 72 dB (@80 Hz) & 70 dB (@80Hz) & 60 dB (@600Hz) & 63 dB (@80 Hz) \\
\hline CMRR & $85 \mathrm{~dB}$ & $85 \mathrm{~dB}$ & $70 \mathrm{~dB}$ & $80 \mathrm{~dB}$ \\
\hline Current consumption & $22 \mu \mathrm{A}$ & $17.5 \mu \mathrm{A}$ & $47.5 \mu \mathrm{A}$ & $47.5 \mu \mathrm{A}$ \\
\hline Power dissipation & $53 \mu \mathrm{W}\left(@ 2.4 V_{\text {supl }}\right)$ & $41 \mu \mathrm{W}\left(@ 2.4 V_{\text {supl }}\right)$ & $133 \mu \mathrm{W}\left(@ 2.8 V_{\text {supl }}\right)$ & $133 \mu \mathrm{W}\left(@ 2.8 V_{\text {supl }}\right)$ \\
\hline Technology & \multicolumn{4}{|c|}{$0.6 \mu \mathrm{m}$, double poly, triple metal CMOS } \\
\hline $\begin{array}{l}\text { Active/Total Chip } \\
\text { Area }\end{array}$ & \multicolumn{4}{|c|}{$1.4 / 4 \mathrm{~mm}^{2}$} \\
\hline
\end{tabular}

